\title{
Legionella's toolbox of effectors
}

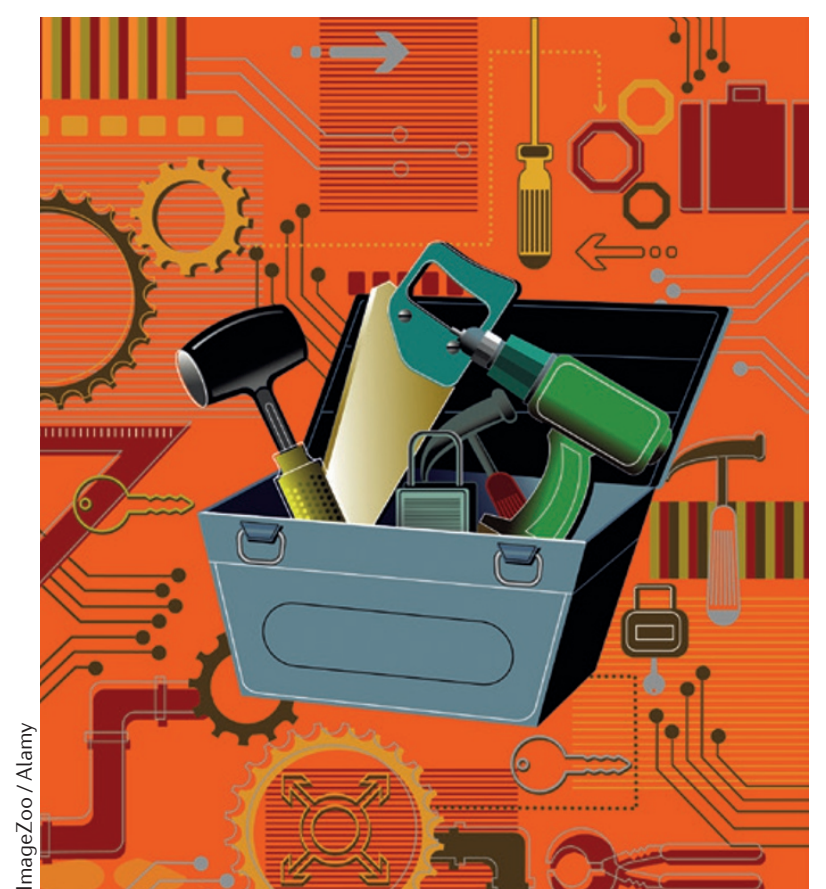

Legionella pneumophila, the causative agent of Legionnaires' disease, relies on a large repertoire of effector proteins to replicate inside host cells. So far, $\sim 300$ effector proteins in L. pneumophila have been identified, but much less is known about effectors in other Legionella species. Burstein et al. sequenced the genomes of 38 different Legionella

\section{Legionella}

can turn

exogenously

acquired genes

into effectors

relatively

quickly

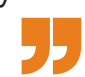

and is present in all Legionella species studied so far; however, with the exception of L. pneumophila, the secreted effectors are mostly unknown. Burstein et al. sequenced and assembled the genomes of isolates from 38 different species and analysed them along three published genomes from L. pneumophila,

Legionella longbeachae and Legionella drancourtii. To identify new effectors, the authors trained a machinelearning algorithm on proteins that were highly similar to known Legionella spp. effectors, taking into account features such as the $\mathrm{Icm} / \mathrm{Dot}$ secretion signal or eukaryotic motifs. Using this algorithm, they identified 5,885 putative effectors; 2,170 of which were novel without orthologues among known effectors.

Surprisingly, only seven effectors were present in all genomes. As these seven effectors had a variable GC content between species, which was similar to the average GC content of the respective genomes, and as their phylogenetic tree corresponded with the species tree, they were probably present in an early ancestor and have evolved in the different species for a long time. Only two of these core effectors reduced growth in deletion mutants, indicating functional redundancy. Nevertheless, the universal presence and evolutionary conservation of the core effectors emphasizes their importance. Interestingly, two of these effectors were also found in distantly related bacteria, probably as a result of cross-genera horizontal gene transfer. Many effectors were present in only one species, and every analysed genome contained at least one such species-specific effector. Interestingly, the GC content of species-specific effectors was lower than the genomic GC content, which suggests that they were recently acquired, maybe from amoeba hosts, the genomes of which generally have a low GC content. This indicates that Legionella spp. can turn exogenously acquired genes into effectors relatively quickly.

The large set of putative effectors enabled Burstein et al. to analyse conserved effector domains, many of which were of eukaryotic origin. Some of the domains, such as phosphatidylinositol-4-phosphatebinding domains, which direct proteins to the Legionella-containing vacuole, or ankyrin-repeats, which mediate protein interactions, are functionally well characterized. Overall, domains were shared between many effectors and occurred in various combinations, which indicates that domain-shuffling has contributed to effector evolution and variability.

The diverse effector repertoire identified in the different Legionella species reflects a high degree of evolutionary plasticity, with the constant acquisition and adaptation of genetic material from host cells or co-infecting pathogens supporting Legionella virulence.

Ursula Hofer

ORIGINAL ARTICLE Burstein, D. et al. Genomic analysis of 38 Legionella species identifies large and diverse effector repertoires. Nat. Genet. http://dx.doi.org/10.1038/ng.3481 (2016) FURTHER READING Escoll, P. et al. Targeting of host organelles by pathogenic bacteria: a sophisticated subversion strategy. Nat. Rev. Microbiol. 14, 5-19 (2015) 